\title{
INSECTOS PERFORADORES DE LAS TRECE MADERAS MÁS COMERCIALIZADAS EN EL VALLE DE ABURRÁ (ANTIOQUIA, COLOMBIA)
}

\author{
Damaging insects of the thirteen more commercialized woods \\ in the Aburrá Valley (Antioquia, Colombia)
}

Palabras clave: albura, duramen, industria de madera, Scolytinae, Bostrichidae, Lyctidae, manejo de maderas.

Keywords: sapwood, heartwood, wood industries, Scolytinae, Bostrichidae, Lyctidae, wood management.

\section{RESUMEN}

Los organismos xilófagos y xilomicetófagos pueden causar un gran deterioro a las maderas. En este estudio se determinó la presencia y se identificaron los insectos que atacan las 13 maderas más comercializadas en el Valle de Aburrá, se determinaron cuáles son los que generan los mayores daños y se identificó el tejido de la madera más atacado. Así mismo, se realizaron observaciones sobre el manejo y las condiciones de almacenamiento de las maderas en 27 industrias madereras en las que se inspeccionaron 426 muestras. Los más insectos comunes y de mayor importancia fueron Scolytinae y Bostrichidae. En el $41 \%$ de las muestras analizadas fue posible identificar el tejido de la madera atacado y aun cuando todas las especies estudiadas presentaron ataques, no se extrajeron muestras de insectos de todas ellas. En general se observaron deficiencias en la manipulación y el almacenamiento de las maderas en las industrias visitadas, lo cual puede aumentar el impacto de los agentes de deterioro.

\begin{abstract}
Xylophagous and xylomicetophagous insects can cause serious deterioration of timber. The insects attacking the thirteen most traded timbers in the Valle de Aburrá were identified and their presence
\end{abstract}

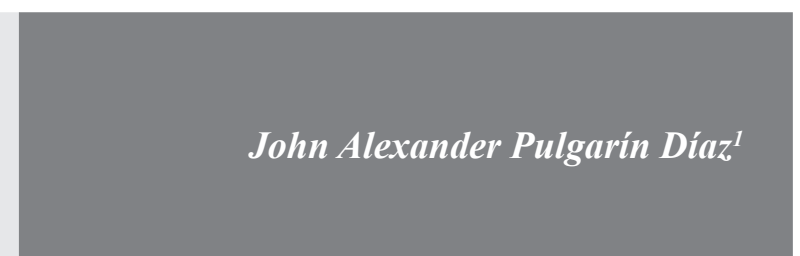

was confirmed. Insects causing most of the damage were determined and the wood tissue most attacked was characterized. Comments on the handling and storage conditions of the wood were recorded in 27 wood industries visited and 426 wood samples were inspected. The most important and most common insects found belong to the Scolytinae and Bostrichidae subfamilies. In addition, the most attacked wood tissue was identified in $41 \%$ of the samples. Even though all species studied showed traces of damage caused by insects, insect samples were not recovered from each wood sample. In general, there were deficiencies in wood handling and storage at the visited industries, which may increase the impact of decay agents.

\section{INTRODUCCIÓN}

La tala de los bosques naturales ha alcanzado niveles críticos (Wilson 1994, FAO 2001, Ministerio de Ambiente, Vivienda y Desarrollo Territorial 2001, CBD 2006) que ponen en riesgo alto la biodiversidad (Wheeler et al. 2004, DNP 2006). Las principales causas en Colombia son la expansión de la frontera agrícola, la explotación maderera y los incendios forestales (DNP 2006).

En una explotación maderera típica se talan los árboles y se envían los productos a los centros de acopio, donde son transformados en bienes de con-

1 Ingeniero Forestal, Candidato a Magister en Ciencias-Entomología, Universidad Nacional de Colombia, sede Medellín. Correspondencia: japulga0@unal.edu.co 
sumo. En este proceso una gran porción del material se descarta por ataques de insectos y hongos (Déon 1990). Abreu et al. (2002) y Sales-Campos et al. (2000) reportan pérdidas cercanas al $60 \%$ en la Amazonía brasilera por el ataque de estos organismos. Según Nair (2007) el desperdicio resultante es enorme aunque no hay estimaciones confiables.

El aprovechamiento racional de las maderas hace necesario mejorar su conocimiento (Cavers et al. 2003) y el de los organismos xilófagos asociados a ellas (Morón 1985). Los insectos xilófagos en Colombia han sido poco estudiados y sus daños no se han cuantificado. Se conocen listados de los insectos asociados a especies forestales de interés económico (Gallego \& Vélez 1992, Madrigal 2002, 2003), aspectos taxonómicos (Bellamy 1995, Martins \& Galileo 2002a , Martins \& Galileo 2002b, QuirozGamboa \& Sepulveda-Cano 2008) y aspectos de biología y comportamiento de algunas especies (Mejía 1997, Gómez \& Madrigal 2001, Aguilar \& Mejía 2004). Solamente un trabajo aborda la sistemática y ecología (Martínez 2000).

El objetivo de este trabajo fue evaluar la ocurrencia y frecuencia de daños causados por insectos, determinar la presencia e identificar las especies insectiles que atacan las 13 maderas más comercializadas en el Valle de Aburrá, así como determinar cuáles insectos generan los mayores daños en las maderas, relacionar su presencia con la parte del tejido atacado (albura o duramen) y realizar observaciones sobre condiciones de almacenamiento de las maderas en las industrias madereras del Valle de Aburrá.

\section{METODOLOGÍA}

El proyecto se desarrolló entre septiembre y octubre de 2004 en industrias madereras ubicadas en los municipios de Envigado, Medellín y Bello, pertenecientes al Área Metropolitana del Valle de Aburrá (Antioquia, Colombia), con un rango de elevación de 1450 a $1575 \mathrm{msnm}, 21-22^{\circ} \mathrm{C}$ de temperatura y $1500 \mathrm{~mm}$ de precipitación promedio anual (Corantioquia 1997).

Se seleccionaron 13 de las especies de mayor frecuencia de ocurrencia en aserríos y depósitos en la región de estudio (Ramírez \& Vásquez 2002). Éstas fueron: abarco (Cariniana pyriformis Miers), carrá (Huberodendron patinoi Cuatrec.), cedro (Cedrela odorata L.), cedro güino (Carapa guianensis Aubl.), chingalé (Jacaranda copaia (Aubl.) D. Don), ciprés (Cupressus lusitanica Mill), nazareno (Peltogyne paniculata Benth.), nogal (Cordia alliodora (Ruiz \& Pav.) Oken), pino pátula (Pinus patula Schiede \& Deppe), roble (Tabebuia rosea (Bertol.) A. DC.), sapán (Clathrotropis brachypetala (Tul.) Kleinhoonte), teca (Tectona grandis L. F.) y tolúa (Pachira quinata (Jacq.) W.S. Alverson).

Se visitaron 27 industrias madereras en las que se inspeccionaron 426 muestras seleccionadas al azar (mínimo 25 por especie), en las cuales se identificaron signos y daños causados por insectos. Las muestras fueron piezas de madera de $20 \mathrm{~cm}$ x $20 \mathrm{~cm}$ x $3 \mathrm{~m}$, aproximadamente, a las que se les cortaron las partes afectadas para llevarlas en bolsas plásticas (Lunz \& Carvalho 2002, Trevisan et al. 2007) al Museo Entomológico Francisco Luis Gallego (MEFLG) de la Universidad Nacional de Colombia, sede Medellín. Se indagó por los tratamientos dados a las maderas y se realizaron observaciones sobre su manejo. De las especies forestales tolúa y carrá no se encontraron muestras suficientes en los locales visitados, por lo que fue necesario colectar piezas en talleres donde se trabajan. Se colectaron nueve y diez respectivamente.

Se midió el contenido de humedad $(\mathrm{CH})$ por el método de desecación al horno a $103 \pm 2^{\circ} \mathrm{C}$ hasta obtener peso constante de una parte de la muestra. Los insectos presentes en las muestras se extrajeron con formón y mazo (Lunz \& Carvalho 2002, Trevisan et al. 2007). Las piezas de madera que contenían insectos inmaduros se ensamblaron con bandas elásticas y se colocaron en recipientes transparentes tapados con tul, tratando de conservar las condiciones de humedad y temperatura apropiadas para su desarrollo.

La determinación de los insectos se realizó hasta la máxima categoría taxonómica posible en el MEFLG por comparación con el material depositado en esa colección y las claves publicadas por Fisher (1950), Arnett (1960) y Wood (2007). La identificación del 
agente que causó el daño de las maderas donde no se encontraron insectos se realizó por comparación con las descripciones encontradas en la literatura disponible (Deangelis 1995, Randall 2000, Ibach 2005) y con las marcas dejadas por los insectos determinados.

La diferenciación entre la albura y el duramen se realizó por observación directa de la coloración de los tejidos. En pátula y ciprés se aplicaron las siguientes soluciones mezcladas en partes iguales antes de realizar la prueba: $2.5 \mathrm{~g}$ de bencidina, 12.5 g de ácido clorhídrico al 25\% y 435 g de agua, más nitrito sódico al 10\% (Berón et al. 1985), para teñir el duramen de rojo oscuro y la albura de amarillo.

Partiendo de los datos obtenidos y con la ecuación 1 se calculó el tamaño de la muestra necesario para tener un error de muestreo menor a 0.05 (Zar 1999).

$$
n=\frac{4 p q}{E^{2}}
$$

Ecuación 1. Donde: $\mathrm{n}=$ tamaño de la muestra necesario para estimar la proporción; $\mathrm{E}=$ límite de error; $\mathrm{p}=$ proporción de elementos con la característica deseada; $\mathrm{q}=1-\mathrm{p}$.

\section{RESULTADOS}

\section{FRECUENCIA DE DAÑOS}

En las 13 especies forestales estudiadas se observaron daños causados por insectos (Tabla 1). Sola- mente en el $15.5 \%$ de las muestras se encontraron insectos. En algunas piezas afectadas se recuperaron insectos adultos o inmaduros y solamente en una muestra de cedro güino se encontró ataque por más de un agente causal.

De acuerdo con el cálculo de error de muestreo (ecuación 1) se encontró que la tolúa tiene el máximo error de muestreo y el nazareno y el sapán el mínimo (Tabla 1). Los valores del límite del error para nazareno, nogal, teca, sapán y pino pátula son los valores más bajos; para ciprés, roble, carrá y abarco se pueden considerar tolerables, y para cedro, chingalé, cedro güino y tolúa los valores de este factor fueron los más altos (Tabla 1).

El tamaño de la muestra usado en este estudio resultó ser menor al estimado para tener un nivel de confiabilidad del 95\% (E=0.05). En la Tabla 1 se observa que para obtener un límite del error de 0.05 y manteniendo la menor proporción de muestras atacadas (0.0286) se necesitarían 45 muestras de cada madera.

\section{DAÑOS E INSECTOS ENCONTRADOS}

Todas las especies insectiles encontradas que causan daño a las maderas pertenecen al orden Coleoptera, con excepción las termitas (Isoptera) observadas en el roble (Figura 1). Es importante notar que en el $78.7 \%$ de las muestras atacadas se encontraron túneles de Scolytinae (Curculionidae, que incluye a Platypodini) comúnmente llamados coleópteros ambrosiales (Figura 1).

Tabla 1. Frecuencia de ataques observados en las muestras de madera obtenidas en depósitos de madera y aserríos en el Valle de Aburrá.

\begin{tabular}{llccc}
\hline Especie forestal & $\mathbf{N}$ & $\begin{array}{c}\text { Porcentaje de muestras } \\
\text { atacadas }\end{array}$ & $\begin{array}{c}\text { Límite del error (E)* } \\
\text { n para E=0.05* }\end{array}$ \\
\hline \hline Abarco (Cariniana pyriformis Miers) & 42 & 28.57 & 0.14 & 326.53 \\
Cedro (Cedrela odorata L.) & 28 & 21.43 & 0.16 & 269.39 \\
Chingalé (Jacaranda copaia (Aubl.) D. Don) & 30 & 46.67 & 0.18 & 398.22 \\
Ciprés (Cupressus lusitanica Mill) & 35 & 8.57 & 0.09 & 125.39 \\
Nazareno (Peltogyne paniculata Benth.) & 35 & 286 & 0.06 & 44.41 \\
Pino pátula (Pinus patula Schiede \& Deppe) & 28 & 3.57 & 0.07 & 55.10 \\
Roble (Tabebuia rosea (Bertol.) A. DC.) & 45 & 13.33 & 0.10 & 184.89 \\
\hline
\end{tabular}




\begin{tabular}{llccc}
\hline Especie forestal & N & $\begin{array}{c}\text { Porcentaje de muestras } \\
\text { atacadas }\end{array}$ & $\begin{array}{c}\text { Límite del error (E)* } \\
\mathbf{n} \text { para E=0.05* }\end{array}$ \\
\hline \hline Teca (Tectona grandis L. F.) & 27 & 3.70 & 0.07 & 57.06 \\
Tolúa (Pachira quinata (Jacq.) W.S. Alverson) & 17 & 23.53 & 0.21 & 287.89 \\
Sapán (Clathrotropis brachypetala (Tul.) Kleinhoonte) & 48 & 4.17 & 0.06 & 63.89 \\
Carrá (Huberodendron patinoi Cuatrec.) & 20 & 5.00 & 0.10 & 76.00 \\
Cedro guino (Carapa guianensis Aubl.) & 25 & 36.00 & 0.19 & 368.64 \\
Nogal (Cordia alliodora (Ruiz \& Pav.) Oken) & 27 & 3.70 & 0.07 & 57.06 \\
\hline Promedio & 31.31 & 15.47 & 178.04 \\
\hline
\end{tabular}

n: tamaño de la muestra calculada con la ecuación 1.

* Ver ecuación 1 .

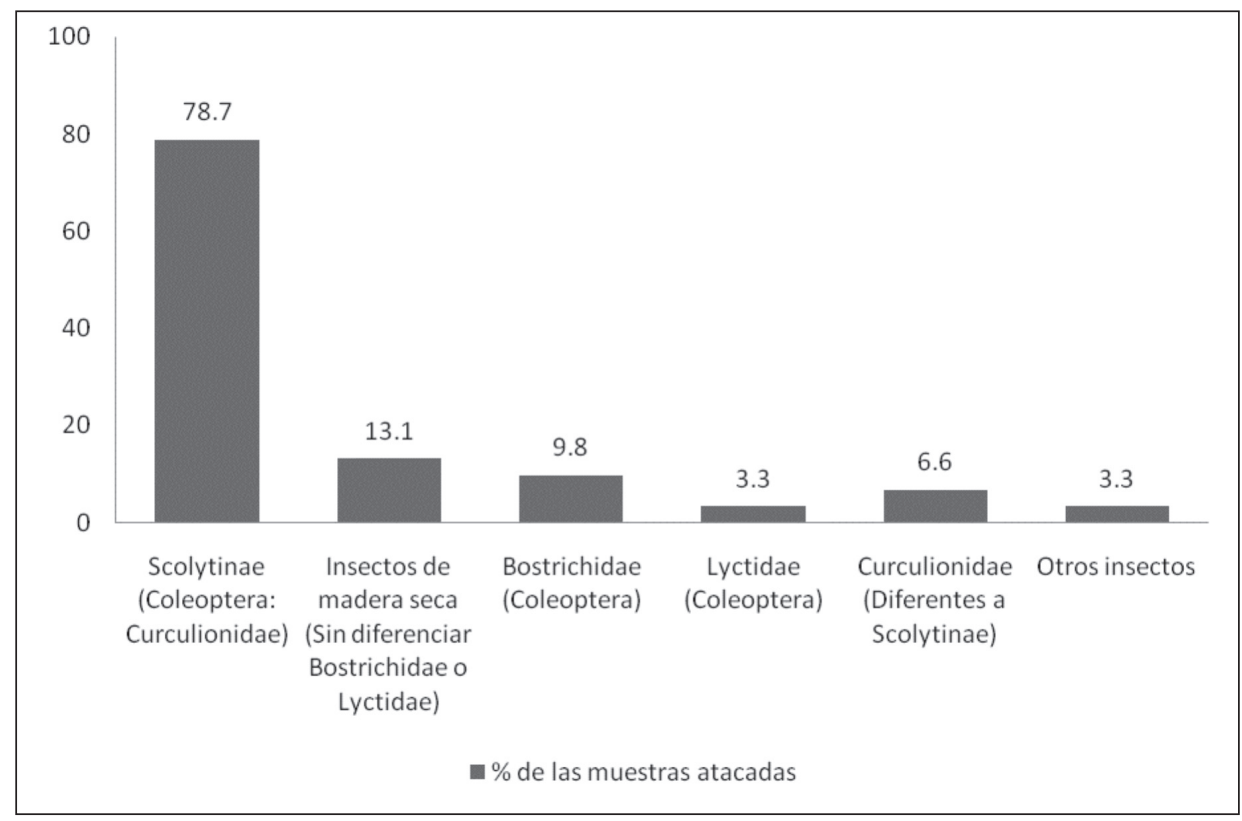

Figura 1. Frecuencia de daños según su agente causal.

En el $41 \%$ de las 61 muestras atacadas se identificó claramente el tejido de madera que fue atacado; se encontró una mayor cantidad de muestras atacadas en la albura (30.88\%) que en el duramen (13.23\%).

En seis muestras se encontraron insectos que no se relacionan con daños a las maderas: Tarsostenus univittatus Rossi (Coleoptera: Cleridae), Solenopsis sp. (Hymenoptera: Formicidae) y un Nitidulidae (Coleoptera).

\section{MANEJO DADO A LAS MADERAS EN LAS INDUSTRIAS VISITADAS}

En general el almacenamiento de las maderas se realizaba en pilas en contacto con el suelo, sin separadores, rodeadas por desperdicios de madera atacados por insectos xilófagos y acumulaciones de agua. En dos lugares visitados las maderas se hallaron a la intemperie. En tres se permitía la entrada de agua lluvia y su encharcamiento. En la mayoría de sitios el secado de las maderas se realizaba 
dejando la madera en el lugar de almacenamiento; solamente dos utilizaban hornos de secado.

En dos de los 27 establecimientos visitados se realizaban tratamientos contra insectos. En uno de ellos se asperjaba esporádicamente ACPM sobre las maderas y en otro, diferentes productos no especificados cuando la proliferación de insectos era alta. Se evidenció la falta de cuidado del estado sanitario del material con el hallazgo de una colonia de termitas (Nasutitermes columbicus Holmgren (Thorne \& Noirot, 1982)) (Isoptera: Termitidae) con obreras desplazándose por las maderas.

\section{DISCUSIÓN}

De acuerdo con la estimación del tamaño de muestra, para obtener una mejor aproximación a la proporción de maderas atacadas y para que la probabilidad de tomar una muestra atacada no sea mayor a 0.05 es necesario aumentar su tamaño.

Los coleópteros de ambrosía (Scolytinae) fueron, en este estudio, los insectos causantes de la mayor cantidad de daños, seguidos en menor proporción por los Bostrichidae. La ausencia de escarabajos ambrosiales en algunas muestras que presentaban sus galerías puede atribuirse a que estos insectos abandonan la madera al disminuir el $\mathrm{CH}$ en busca de material con las condiciones necesarias para el desarrollo de su progenie.

Entre los insectos xilófagos, los coleópteros constituyen el grupo que causa las mayores pérdidas en plantaciones forestales, centros de acopio y transformación de la madera (Flechtmann \& Gaspareto 1997, Madrigal 2003, Walker 2006); de éstos, los más importantes económicamente son los Scolytinae (Madrigal 2003). En países de tradición forestal se reportan diferentes Scolytinae que matan a su hospedero y/o atacan madera apeada causando millonarias pérdidas (Pureswaran \& Borden 2005, Salle et al. 2005, Yan et al. 2005, Liu \& Dai 2006, Rice et al. 2008). Su ataque causa grandes pérdidas comerciales en Colombia al reducir la calidad de la madera, pero no se encuentra en la literatura una medida.

Tabla 2. Insectos encontrados, insectos perforadores de las maderas estudiadas y tejido atacado.

\begin{tabular}{|c|c|c|c|}
\hline $\begin{array}{l}\text { Especie arbórea y } \\
\text { cantidad de muestras } \\
\text { con ataque }\end{array}$ & $\begin{array}{l}\text { Insectos encontrados } \\
\text { y frecuencia absoluta }\end{array}$ & $\begin{array}{l}\text { Insecto perforador } \\
\text { y frecuencia relativa } \\
\text { de ataque }\end{array}$ & $\begin{array}{l}\text { Porcentaje de ataque } \\
\text { según el tejido (albura y } \\
\text { duramen)* }\end{array}$ \\
\hline Carapa guianensis (9) & $\begin{array}{l}\text { Xyleborus ferrugineus (F.) (Coleoptera: } \\
\text { Scolytinae) (4) }\end{array}$ & $\begin{array}{l}\text { Scolytinae (88.8\%), Bostrichidae } \\
(11.11 \%) \text {, otros (11.11\%) }\end{array}$ & Du: $11 \%$ \\
\hline Cariniana pyriformis (12) & $\begin{array}{l}\text { Xyleborus sp. (2), X. ferrugineus (11) y X. } \\
\text { affinis Eichhoff (4) }\end{array}$ & Scolytinae (100\%) & Al: $41.6 \%$ Du: $25 \%$ \\
\hline Cedrela odorata (6) & $\begin{array}{l}\text { Prostephanus arizonicus (Coleoptera: } \\
\text { Bostrichidae) (7) }\end{array}$ & Scolytinae (50\%), Bostrichidae (50\%) & Al: $16 \%$ Du: $16 \%$ \\
\hline Clathrotropis brachypetala (2) & $\begin{array}{l}\text { Tarsostenus univittatus Rossi (Coleoptera, } \\
\text { Cleridae) (1), Minthea sp. (Coleoptera, } \\
\text { Lyctidae) (3), Xyleborus ferrugineus (1) }\end{array}$ & Scolytinae (50\%), Lyctidae (50\%) & Al: $100 \%$ Du: $50 \%$ \\
\hline Cordia alliodora (1) & & Scolytinae (100\%) & Al: $100 \%$ \\
\hline Cupressus lusitanica (3) & & Curculionidae (100\%) & Al: $100 \%$ \\
\hline Huberodendron patinoi (1) & Nitidulidae (Coleoptera) (1) & Scolytinae (100\%) & \\
\hline
\end{tabular}




\begin{tabular}{|c|c|c|c|}
\hline $\begin{array}{l}\text { Especie arbórea y } \\
\text { cantidad de muestras } \\
\text { con ataque }\end{array}$ & $\begin{array}{l}\text { Insectos encontrados } \\
\text { y frecuencia absoluta }\end{array}$ & $\begin{array}{l}\text { Insecto perforador } \\
\text { y frecuencia relativa } \\
\text { de ataque }\end{array}$ & $\begin{array}{l}\text { Porcentaje de ataque } \\
\text { según el tejido (albura y } \\
\text { duramen)* }\end{array}$ \\
\hline $\begin{array}{l}\text { Huberodendron patinoi } \\
(11)^{* *}\end{array}$ & $\begin{array}{l}\text { Lyctus brunneus (Stephens) (Coleoptera, } \\
\text { Lyctidae) (1) }\end{array}$ & $\begin{array}{l}\text { Otros }(45.45 \%), \text { Scolytinae }(45.45 \%), \\
\text { Lyctidae }(9.09 \%)\end{array}$ & \\
\hline Jacaranda copaia (14) & $\begin{array}{l}\text { Euplatypus sp. (Coleoptera: Scolytinae) } \\
\text { (14), Tarsostenus univittatus (2), } \\
\text { Xyleborus sp. (1), X. affinis (2), X. } \\
\text { ferrugineus (5) }\end{array}$ & $\begin{array}{l}\text { Scolytinae (78.5\%), Bostrichidae } \\
\text { (14.28\%), Lyctidae (7.14\%) }\end{array}$ & Al: $50 \%$ \\
\hline Peltogyne paniculata (1) & & Scolytinae (100\%) & Al: $100 \%$ \\
\hline Pachira quinata (4) & $\begin{array}{l}\text { Xyleborus sp. (1), X. ferrugineus (3) y X. } \\
\text { affinis (4) }\end{array}$ & Scolytinae (100\%) & Al: $50 \%$ Du: $25 \%$ \\
\hline Pachira quinata $(9)^{* *}$ & & Scolytinae (77.7\%), otros (22.2\%) & Al: $66 \%$ Du: $44 \%$ \\
\hline Pinus patula (1) & $\begin{array}{l}\text { Hexarthrum sp. (Coleoptera: } \\
\text { Curculionidae) (5) }\end{array}$ & Curculionidae (100\%) & Al: $100 \%$ \\
\hline Tabebuia rosea (6) & Nitidulidae (1) Xyleborus ferrugineus (2) & Scolytinae (83.3\%), Isoptera (16.7\%) & \\
\hline Tectona grandis (1) & $\begin{array}{l}\text { Solenopsis sp. (Hymenoptera: } \\
\text { Formicidae) (1) }\end{array}$ & Scolytinae (100\%) & Al: $100 \%$ \\
\hline
\end{tabular}

Los coleópteros de los géneros Xyleborus y Euplatypus atacan principalmente madera (albura y duramen) con $\mathrm{CH}$ superior al 48\% (Coulson \& Witter 1985, USDA 1985), generando deterioro severo del material (Madrigal 2003). En la mayoría de los casos estos barrenadores prefieren árboles debilitados, aunque en situaciones de explosión demográfica atacan árboles sanos. Los hábitos y comportamiento de $X$. affinis son bastante similares a los de $X$. ferrugineus y $X$. volvulus (Fabricius) (Wood 2007). X. ferrugineus puede destruir completamente la albura de las trozas en campo o en aserrío, constituyéndose en la especie más destructiva en áreas reforestadas de Suramérica (Wood 2007).

Los bostríquidos atacan árboles sanos y débiles, prefiriendo los últimos, y se reproducen dentro de la madera seca hasta convertirla en polvo (Madrigal 2003). El ataque de estos insectos se da en maderas con $\mathrm{CH}$ igual o menor a $30 \%$, atacando solamente la albura. En la literatura solo se registra el primer reporte de Prostephanus arizonicus Fisher en Arizona, EeUU, (Fisher 1950).

Numerosas especies de la familia Lyctidae, entre ellas especies de los géneros Lyctus y Minthea, han sido ampliamente distribuidas por medio del comercio, los que poseen gran capacidad para destruir madera seca en muy poco tiempo, prefiriendo la albura por su mayor concentración de almidón (USDA 1985).

Las diferentes especies de Cossoninae (Coleoptera: Curculionidae) atacan la madera con diferentes $\mathrm{CH}$, prefiriendo la albura. Algunas especies de la subfamilia, incluidas varias del género Hexarthrum, atacan madera instalada con bajo CH (USDA 1985).

Al parecer los individuos de Tarsostenus univittatus que se encontraron dentro de dos de las tres muestras colocadas en las cámaras de cría, depredaron los insectos que se pretendieron criar. Es posible que T. univittatus sea un importante controlador de 
insectos xilófagos, ya que USDA (1985) registra a este clérido como consumidor importante de Lyctidae y Bostrichidae.

Aun cuando se encontró alta dificultad para diferenciar la albura del duramen en algunas piezas de madera, en este estudio se encontraron más ataques en la albura, posiblemente debido a que posee menor concentración de extractivos tóxicos y mayor de azúcares y proteínas que el duramen (Taylor et al. 2002, Wiedenhoeft \& Miller 2005, Walker 2006). De esta forma, el duramen que se encontró atacado pudo haber sido obtenido de árboles jóvenes o con agresiones previas de organismos filófagos o con sitios pobres (Compte \& Caminero 1982).

Según el USDA (1985) los escarabajos xilomicetófagos (géneros Xyleborus, Euplatypus y Platypus) sólo perforan la albura, sin embargo en este estudio se encontró ataque en el duramen, que coinciden con los reportes de Furniss \& Carolin (1977). En contraste, a pesar de que los Lyctidae y Bostrichidae pueden atacar tanto albura como duramen (USDA 1985, Déon 1990, Ibach 2005), en el presente estudio no se encontró ataque en el duramen.

\section{DAÑOS ENCONTRADOS EN LAS 13 ESPECIES ESTUDIADAS}

Los daños observados en las maderas en las que no fue posible identificar directamente el agente causal probablemente fueron realizados por algunos de los insectos relacionados en la Tabla 3 y/o por los aquí registrados, ya que los insectos xilófagos tienen poca especificidad de hospedero (Novotny $\&$ Basset 2005). Los nitidúlidos encontrados en algunas muestras de carrá se alimentan de hongos (Furniss \& Carolin 1977), savia fermentada y exudados de savia de trozas recién cortadas (USDA 1985); no se encuentran reportes de daños de estos insectos en maderas.

Tabla 3. Registros encontrados en la literatura sobre insectos perforadores de las maderas incluidas en el presente estudio.

\begin{tabular}{|c|c|c|}
\hline Especie forestal & Especie de insecto & Autor \\
\hline Carapasp. & $\begin{array}{l}\text { Sphaerotrypes grandis Schedl, } 1957 \text { (Curculionidae: } \\
\text { Scolytinae) }\end{array}$ & Bright \& Skidmore (1997) \\
\hline $\begin{array}{l}\text { Carapa guianensis y Cedrela } \\
\text { odorata }\end{array}$ & Xyleborus affinis Eichhoffy Platypus paralellus (F.) & Abreu et al. (2002) \\
\hline Carapa procera D.C. & $\begin{array}{l}\text { Polygraphus granulatus Eggers, 1932, P. granulifer Eggers, } \\
1935 \text { (Curculionidae: Scolytinae) }\end{array}$ & Bright \& Skidmore (1997) \\
\hline Cedrela odorata & $\begin{array}{l}\text { Colydiidae, Curculionidae, Scarabaeidae y Scolytinae } \\
\text { (Coleoptera) }\end{array}$ & Abreu \& Rodigues (1989) \\
\hline Cedrela odorata & Xyleborus ferrugineus (Fabr.) & Madrigal (2002) \\
\hline Cedrela odorata & $\begin{array}{l}\text { Xyleborus affinis Eichhoff, X. ferrugineus (F.) y X. volvulus } \\
\text { (F.) }\end{array}$ & $\begin{array}{l}\text { Abreu (1992), Bright \& Skidmore } \\
\text { (1997) }\end{array}$ \\
\hline Cedrela odorata & Derobrachus sp. (Coleoptera: Cerambycidae) & Madrigal (2003) \\
\hline Cordia sp. & $\begin{array}{l}\text { Theoborus micarius Wood, Xyleborus tolimanus Eggers, X. } \\
\text { pseudotenuis Scheldl. (Curculionidae: Scolytinae) }\end{array}$ & Wood et al. (1991) \\
\hline Cupressus /usitanica & Anchonus sp. (Coleoptera: Curculionidae) & $\begin{array}{l}\text { Gallego \& Vélez (1992) Madrigal } \\
\text { (2003) }\end{array}$ \\
\hline Jacaranda copaia & $\begin{array}{l}\text { Colydiidae, Curculionidae, Staphylinidae y Scarabaeidae } \\
\text { (Coleoptera) }\end{array}$ & Abreu \& Dietrich (1989) \\
\hline Jacaranda copaia & Coptotermes sp. (Isoptera: Rhinotermitidae) & Jesús et al. (1998) \\
\hline Jacaranda copaia & Hepialidae (Insecta: Lepidoptera) & Gómez \& Madrigal (2001) \\
\hline
\end{tabular}




\begin{tabular}{lll}
\hline Especie forestal & \multicolumn{1}{c}{ Especie de insecto } & Autor \\
\hline Pachira quinata & Xyleborus ferrugineus (F.) y Platypus sp. & Madrigal (2003) \\
Tabebuia rosea & Hypothenemus sp. (Coleoptera: Scolytinae) & Madrigal (2003) \\
Tectona grandis & Xyleborus morigerus Blandford. & Nair (2001) \\
Tectona grandis & $\begin{array}{l}\text { Dactylipalpus transversus Chapuis, 1869 (Coleoptera: } \\
\text { Scolytinae) }\end{array}$ & Bright \& Skidmore (1997) \\
\hline
\end{tabular}

Otros grupos insectiles observados como Nannotrigona pachysoma, en una troza de teca, corresponden posiblemente a la colonización de una cavidad encontrada por las abejas. Según Nates-Parra (1990), las abejas de este género nidifican en cualquier cavidad disponible. La presencia de un individuo del género Solenopsis en túneles de escarabajos ambrosiales en teca posiblemente esté relacionada con la búsqueda de alimento. IAvH (1999) reporta algunas especies de este género como omnívoras; Martínez et al. (2002) las registran como predadoras de otros insectos.

A pesar de la gran importancia comercial que tienen las maderas de nazareno, carrá, sapán, chingalé y abarco, no se encontraron reportes de ataques por insectos.

\section{MANEJO DADO A LAS MADERAS EN LAS INDUSTRIAS MADERERAS VISITADAS}

El encharcamiento de agua en los locales donde se manejan las maderas aumenta el $\mathrm{CH}$ de las maderas y favorece la proliferación de organismos xilófagos (como hongos y escolítinos), lo cual constituye una fuente permanente de inóculo para las piezas de maderas nuevas (Flechtmann \& Gaspareto 1997, Highley 1999).

Las condiciones de almacenamiento de las maderas observadas en este estudio coinciden con reportes de Manaos y de la misma zona de estudio, donde se propician condiciones para el ataque de organismos xilófagos que perjudican la calidad del material (Sales-Camposet al.2000, Abreuet al.2002). Los primeros autores reportan que pocos aserríos hacen uso de secado artificial, mientras que en el Valle de Aburrá el 20\% de las industrias madereras secan el material al aire y el $10 \%$ en hornos (Ramírez \& Vásquez 2002). Así mismo, el hallazgo de insectos xilófagos en las maderas de los aserríos de Manaos demuestra serias deficiencias en su manejo, derivando en pérdidas del $40 \%$ del material (Sales-Campos et al. 2000) o del 60\% en las industrias madereras de la región amazónica brasilera (Abreu et al. 2002). Así mismo, mientras que Ramírez \& Vásquez (2002) afirman que solo el $7 \%$ de los aserríos y depósitos de madera del Valle de Aburrá usan tratamientos inmunizantes, SalesCampos et al. (2000) y Abreu et al. (2002) reportan que ninguno de los aserríos encuestados usa tales tratamientos.

Usar secadores artificiales y evitar humedades en los depósitos de las maderas son factores cruciales para evitar que los Scolytinae que vienen dentro de las maderas desde el bosque, continúen perforando el material, por tanto se recomienda realizar esfuerzos en este sentido.

\section{CONCLUSIONES}

De las especies más comercializadas en el Valle de Aburrá, el chingalé fue la especie con mayor proporción de muestras atacadas, seguida en orden por el cedro güino, el abarco, la tolúa y el cedro. A su vez, los insectos responsables de la mayor cantidad de daños son especies de la subfamilia Scolytinae y de la familia Bostrichidae. En este estudio se evidenció el inadecuado tratamiento dado a las maderas en las industrias madereras visitadas, lo que puede aumentar la incidencia de organismos que degradan la madera y causan pérdidas económicas. Es necesario realizar estudios que demuestren la existencia de pérdidas de dinero importantes para enfrentar el problema desde la economía. 
Para las condiciones en las que se desarrolló la presente investigación es necesario un tamaño de muestra mayor a 32 en promedio para todo el muestreo, para conocer con mayor certeza los insectos xilófagos que atacan las maderas estudiadas y su impacto.

\section{AGRADECIMIENTOS}

El autor agradece a Corantioquia por la financiación; al Departamento de Ciencias Forestales y a los laboratorios de Entomología y de Productos Forestales de la Universidad Nacional de Colombia, sede Medellín, por su apoyo logístico, y al profesor Gilberto Morales Soto por su valiosa colaboración en la corrección del documento final.

\section{REFERENCIAS BIBLIOGRÁFICAS}

Abreu, R. L. S. 1992. Estudo da ocorrência de Scolytidae e Platypodidae em madeiras da Amazônia. Acta Amazonica 22 (3): 413-420.

Abreu, R. L. S. \& C. R. C. Dietrich. 1989. Ocorrência de besouros (Insecta: Coleoptera) em madeiras úmidas. Encontro brasileiro de madeira, 3 San Carlos, Volume 4. Anais USP-EESC. Pp. 227-237.

Abreu, R. L. S., C. Sales-Campos, R. E. Hanada, F. J. Vasconcellos \& J. A. Freitas. 2002. Avaliação de danos por insetos em toras estocadas em indústrias madeireiras de Manaus, Amazonas, Brasil. Revista Árvore 26 (6): 789-796.

Aguilar, C. M. \& L. C. Mejía. 2004. Cría de Minthea squamigera (Coleoptera: Lyctidae) en condiciones de laboratorio. Ingeniera Forestal, Facultad de Ciencias Agropecuarias, Universidad Nacional de Colombia, sede Medellín.Pg. 34.

Arnett, R. H. 1960. The beetles of the United States (a manual for identification). The Catholic University of American Press. Washington, D.C. Pg. 1112.

Bellamy, C. L. 1995. A new species of Chrysobothris Eschscholtz from Parque Nacional de Chimbiriquete, Colombia (Coleoptera: Buprestidae). The Coleopteris Bulletin 49 (2): 191-194.
Berón, C., M. R. Ramírez \& L. C. Mejía. 1985. Evaluación de resistencia del pátula (Pinus patula Schiede \& Schltdl. \& Cham.) y ciprés (Cupressus lusitanica Mill) al ataque de termites. Ingeniería Forestal, Facultad de Ciencias Agropecuarias, Universidad Nacional de Colombia, sede Medellín. Pg. 59.

Bright, D. E. \& R. E. Skidmore. 1997. A cata$\log$ of Scolytidae and Platypodidae (Coleoptera), supplement 1 (1990-1994). NRC Research Press. Ottawa, Ontario. Pg. 368.

Cavers, S., C. Navarro \& A. J. Lowe. 2003. Chloroplast DNA phylogeography reveals colonization history of a neotropical tree, Cedrela odorata L., in Mesoamerica. Molecular Ecology: 1451-1460.

CBD. 2006. Global biodiversity outlook 2 . Convenion on Biological Diversity. Pg. 81.

Compte, A. \& M. Caminero. 1982. Las comunidades de coleópteros xilófagos de las encinas de los alrededores de Madrid. Graellsia 38: 201-217.

Corantioquia. 1997. Plan de gestión ambiental regional 1998-2006. Imprenta Departamental de Antioquia. Medellín. Pg. 495.

Coulson, R. N. \& J. A. Witter. 1985. Forest entomology, ecology and management. Wiley-Interscience. USA. Pg. 669.

Deangelis, J. 1995. Preventing and controlling powderpost beetles in and around the home. A Pacific Northwest Extension Publication. PNW 326: 1-4.

Déon, G. 1990. Manual for the preservation of wood in the tropics. International Tropical Timber Organization and Centre Technique Forestier Tropical, Division of CIRAD, Jouve. Pg. 112.

DNP. 2006. Plan Nacional de Desarrollo 20062010. Bogotá. Pg. 548.

FAO. 2001. The state of the world's forests. Food and Agriculture Organization. Roma, Italia. Pg. 180.

Fisher, W. S. 1950. A revision of the North American species of beetles belonging to the family Bostrichidae. United Department of Agriculture, Miscellaneous Publications 698: 157. 